\title{
Supersymmetric Lie Algebra
}

\author{
Jacob M. Schreiber \\ 5 Sharet street, Ramat - Gan, Israel \\ Tel: 972-3-673-4060 E-mail: ora1963@gmail.com
}

\begin{abstract}
Received: October 31, 2011 Accepted: November 17, 2011 Published: February 1, 2012
doi:10.5539/jmr.v4n1p41 URL: http://dx.doi.org/10.5539/jmr.v4n1p41
\end{abstract}

\begin{abstract}
This work is an investigation into the structure and properties of Lie hypermatrix algebra generated by a semisimple basis. By using new algebraic tools; namely cubic hypermatrices I obtain an algebraic structure associated with the basis of a semisimple Lie algebra, and I show that the semisimple Lie basis is a generator of infinite periodic semisimple hypermatrix structures, that has a classical Lie algebra decomposition (Bourbaki, 1980; Humphreys, 1972; Serre, 1987); specifically a set of Lie algebras composed of hypermatrices. The generators of higher dimensional semisimple Lie algebra are shown to be special supersymmetric, anti-symmetric and certain skew-symmetric hypermatrices.
\end{abstract}

Keywords: Anti-symmetric, Basis, Generator, Hypermatrices, Ideal, Lie algebra, Semicimplicity, Skew-symmetry, Supersymmetry

\section{Introduction to Hypermatrices}

In the literature A. Cayley (1843), and others had investigated hypermatrices, and hyper-determinants, also known as full-sign determinant of higher class, or the p-way matrix and its determinant. Usually the investigation was carried out with respect to all possible slices of the object. In most of this work the object is assumed to be composed of square sheets of matrices, and have the cubic $W n \times n \times n \ldots \times n$ configuration.

The hypermatrices components are all parallel to one plane, e.g. the plane of the page and the matrix sheets are set in a cubic hypermatrix arrangement. All algebraic operations of hypermatrices are carried with respect to that orientation. A good way to visualize it is to think of the nth-cube, and its components, the matrix elements set as $2 \mathrm{~d}$-squares, parallel with respect to the plane of the page, in this paper the fourth cube will suffice. The algebra of these objects is a limited generalization of $M_{n \times n}$ matrix theory, in the algebraic sense, and it turns out to be a good way to obtain some information on the global structure of algebraic higher dimensional cubic matrix arrangements and hypermatrices. These cubic structures tend to resist direct algebraic manipulation with ordinary algebraic tools and there is a need to redefine elementary algebraic operations such as multiplication. The main reason to use cubic structures to investigate hypermatrices is that they posses certain symmetric anti-symmetric and skewsymmetric characteristics that are useful for studying higher dimensional matrix arrangements.

\subsection{Algebraic definitions of hypermatrices and hypermatrix Lie algebra}

\subsubsection{Multiplication of hypermatrices}

Example:For a cubic hypermatrix W composed of two matrices $S_{1}$ and $S_{2}$ set on two faces of a cube here denoted by $W=\left(\begin{array}{l}S_{2} \\ S_{1}\end{array}\right)$, we multiply the hypermatrices $W_{2 \times 2 \times 2}$, and $T_{2 \times 2 \times 2}$ sheet into sheet as we multiply matrices, while maintaining a clockwise direction of multiplication, hence:

$$
W \times T=\left(\begin{array}{l}
S_{2} \\
S_{1}
\end{array}\right) \times\left(\begin{array}{l}
T_{2} \\
T_{1}
\end{array}\right)=\left(\begin{array}{ccc} 
& S_{2} T_{1} & \\
S_{1} T_{2} & & S_{2} T_{2} \\
& S_{1} T_{1} &
\end{array}\right)=W_{2 \times 2 \times 4}^{*}
$$

${ }_{n \times n \times n \ldots}$ is an indication of the dimensional extension of the hyper-matrix.

Definition 1 (Lie Algebra of Hypermetrices) Consider the space $\{W\}$ over a field F, with an operation $W W \in W^{*}$. Denote by $\left(W_{i}, W_{j}\right)$ the hypermatrix Lie bracket over $\mathrm{F}$, which constitutes a Lie hypermatrix algebra if the following conditions are satisfied:

A) $W W \in W^{*}$ where $(W s i, W s j) \in W^{*}, W s i$ a component sheet of $W^{*} k$, i.e., $(W, W) \in\left\{\times,+,-, W^{*}\right\} \in L^{*}\{W\}-$ a linear combination in $W^{*} i$ sheets.

B) 1) the bracket operation is bilinear. 
2) $(W, W)=0^{*}$ for all $W \in\{W\}$.

C) $\left(W_{i},\left(W_{j}, W_{k}\right)\right)+\left(W_{j},\left(W_{k}, W_{i}\right)\right)+\left(W_{k},\left(W_{i}, W_{j}\right)\right)=0^{3 \times *}, \forall W_{i}, W_{j}, W_{k} \in\{W\}$.

The hypermatrix algebra has to be closed in terms of its components, and with respect to the field operations, in the sense that the component sheets $\left\{W_{s i} \in W\right\}$ are well defined in the extended space, under the bracket operation $\{W W \in W *\}$.

Definition 2 A subset $I$ of $W$ is called an ideal of $W$ if $W_{i} \in I, W_{j} \in W$ imply $\left[w_{i}, w_{j}\right] \in I, \forall i, j$.

Definition 3 An hypermatrix Lie algebra $W$ is said to be simple if it is not commutative, and if its only ideals are $\{0\}$ and itself, and if $(W, W) \neq 0$.

Definition 4 The hypermatrix Lie algebra $\{\mathrm{W}\}$ is called semisimple if it has no proper abelian ideals other then $\{0\}$.

\subsection{Clasical semisimpe Lie algebra and hypermatrix Lie algebra}

Trace Zero Algebra: we define the following matrices:

$$
x=\left(\begin{array}{ll}
0 & 1 \\
0 & 0
\end{array}\right), y=\left(\begin{array}{ll}
0 & 0 \\
1 & 0
\end{array}\right), h=\left(\begin{array}{cc}
1 & 0 \\
0 & -1
\end{array}\right)
$$

We have the standard relations with respect to $\mathrm{x}, \mathrm{y}, \mathrm{h}$ :

$<$ Table 1>

By setting the basis elements on the faces of a cube we create the first Lie algebra hypermatrix extension.

\section{The First Extension}

Next we define the following hypermatrices on the front and back of the faces of a 3-cube:

$$
\begin{aligned}
& t_{1}=\left\{\begin{array}{l}
X \\
X
\end{array}\right\}, t_{2}=\left\{\begin{array}{l}
x \\
y
\end{array}\right\}, t_{3}=\left\{\begin{array}{l}
x \\
h
\end{array}\right\} \\
& t_{4}=\left\{\begin{array}{l}
y \\
X
\end{array}\right\}, t_{5}=\left\{\begin{array}{l}
y \\
Y
\end{array}\right\}, t_{6}=\left\{\begin{array}{l}
y \\
h
\end{array}\right\} \\
& t_{7}=\left\{\begin{array}{l}
h \\
X
\end{array}\right\}, t_{8}=\left\{\begin{array}{l}
h \\
y
\end{array}\right\}, t_{9}=\left\{\begin{array}{l}
h \\
h
\end{array}\right\}
\end{aligned}
$$

\subsection{The fourth dimensional cube and the associated hypermatrix Lie algebra}

Using hypermatrix multiplication the following relations are obtained with respect to the trace zero elements $\{T\}=$ $\left\{t_{1}, t_{2}, t_{3}, t_{4}, t_{5}, t_{6}, t_{7}, t_{8}, t_{9}\right\}$, and the $\mathrm{W}$ trace zero elements on four faces, parallel to the page, on a 4 dimensional-cube:

$<$ Figure $1>$

$<$ Table $2>$

We notice the following relations in the table: $y x=\left(\begin{array}{ll}0 & 0 \\ 0 & 1\end{array}\right), x y=\left(\begin{array}{ll}1 & 0 \\ 0 & 0\end{array}\right)$, in short $y x=I-x y, x y=I-y x$.

$x x=0, y y=0, h h=I, y h=y, h y=-y, h x=x, x h=-x, x h-y x=-x-y x=x(-1 I-y x)$.

$<$ Table 3>

From table 2 it follows that a simple cubic hypermatrices Lie algebra of the second order is not semisimple, but it has a subalgebra generated by $\left(t_{1}, t_{5}, t_{9}\right)$ which is composed of symmetric hypermatrices and it is a Lie semisimple type algebra, the larger hypermatrix algebra has trace zero sub algebras and non-trace-zero sub-algebras that will be pointed out in this paper. From table 2 it can also be seen that the bracket operation results in a trace zero element if and only if either the component hypermatrices are: a) super-symmetric, i.e. identical in all plane direction of transposing, b) anti-symmetric: have reversed order of elements, or if, c) super-skewsymmetric in at least one direction. One of the components is zero and the other is a basis element, d) both are symmetric, or identical non-zero and are not basis elements, e) both are zero hypermatrices.

In this article it will be shown that the resulting hypermatrix products are trace zero type if and only if the components matrices of the hypermatrices are arranged symmetrically in the hypermatrices, i.e., the hypermatrices are symmetric in terms of the constituent sub-matrices or that the hypermatrices are arranged skew symmetrically in higher order hypermatrices, or cubic hypermatrices.

Lemma 1 The second order algebra is not a closed algebra (not entirely a semisimple algebra), it is a Lie hypermatrix algebra. If we apply to it the Lie bracket operation It will also be generated into an open Lie algebra. 
Proof: See table 2. Lie products of non symmetric and non antisymmetric hypermatrices have non zero trace.

One of the objectives of this paper is to characterize an hyper-semisimple algebra generated by the semisimple set $\left\{t_{i}\right\} i=$ $1, \ldots, 9$ of order 1 , especially the supersymmetric the symmetric elements and the higher supersymmetric, skewsymmetric and antisymmetric generators of the extended Lie hypermatrix algebra $\left\{\left\{t_{i, j}\right\},\left\{t_{[i, j],[i, j]}\right\}, \ldots\right\}$.

The basic structure of the Lie algebra is maintained in 4-dimensional cubic extension however as seen in table 2 the second order elements generated by $\left\{t_{i}\right\} i=1, \ldots, 9$ are not all trace zero elements so that the algebra is not closed in the semisimple sense. For example, the semisimple set $\left\{t_{i}\right\} i=1, \ldots, 9$ generates non-semisimple elements, such as I- the identity.

\subsection{Elements of order 2 and higher}

In short, we can write the elements of order 2 with a double index, as: $t_{1,1}, t_{5,1}, \ldots, t_{i, j}$. When we generate the next level Lie products, if we continue the process some elements will evolve into trace zero elements, and in higher generations of sub-algebras other elements that were not trace zero elements become trace zero elements under the Lie product. For example: the entry $t_{9,6}$ in table 2 is not a trace zero element yet in the next cubic extension $\left(t_{9,6}, t_{2,4}\right)$ it evolves into a trace zero element of order 3 .

\subsection{Semisimplicity of order 3 and higher}

Using the elements of table 2 it is possible to obtain all elements of order 3 that are generated by the Lie bracket operation, and we may generate the next level in the fourth dimensional cube by taking all possible arrangements of elements of order 2 in sets of 16 elements, arranging them on the 24-sheets (faces) of a 4-dimensional cube. We may generate the next level in the seventh dimensional cube by taking all possible arrangements of elements of order 2 in sets of 256 elements, arranging them on the 672-sheets (faces) of a 7-dimensional cube along sheets angled with respect to the page horizontal plane. This is, in general terms, how the generation of Lie algebra hypermatrices progresses along the space line of hypermatrices.

\subsection{Properties of semisimple hypermetrix algebra}

The set of elements $\left\{t_{1}, t_{5}, t_{9}\right\}$ and its analogous in higher dimensions constitute a fundamental extendable set of nthsemisimple algebra up to a structure constant. It constitute an open semisimple subalgebra $\left\{\left\{t_{i, j}\right\},\left\{t_{[i, j],[i, j]}\right\} \ldots\right\}$ in $\left\{W^{n}, n>\right.$ $2\}$. It has a nilpotent subset, composed of the idempotent elements in $\left\{W^{n}, n>2\right\}$.

The center of a semisimple hypermatrix algebra $\{W\}$ is the set $C=\left\{\left[w_{i}, w_{j}\right] \in W \mid\left[w_{i}, w_{j}\right] \in W_{\text {semisimple }}, \forall i, j\right\}$.

Lemma 2 The center of a semisimple hypermatrix algebra $\{W\}$ is simple.

For any non trivial algebra the center is composed of the structure elements $t_{1}, t_{5}, t_{9}, \ldots \in W$ and their product up to a constant we define an hypermatrix Lie algebra $\mathrm{W}$ as simple if it is not commutative, and if its only ideals are $\{0\}$ and itself, and if $(W, W) \neq 0$. The only ideal I of $t_{1}, t_{5}, t_{9} \ldots \in W$ is the zero ideal.

The elements of the second order set are either nilpotent, supersymmetric, symmetric, skewsymmetric or are trace zero elements, or not trace zero elements.

Generally if we multiply two trace zero elements we obtain a trace zero element. $W \operatorname{Tr}\{0\} \times W \operatorname{Tr}\{0\}=W \operatorname{Tr}\{0\}$, however under the bracket operation the resulting product is not necessarily a trace zero element.

It will be shown that if the sheet structure of the hypermatrices $W_{i}, W_{j}$ is symmetric the resulting bracket operation $\left\{\left[W_{i}, W_{j}\right]\right\} \in\left\{W^{*}\right\}$ must be a trace zero element. Therefore, we have the following theorem.

Theorem 1 The Lie product of symmetric trace zero type special linear $S l_{2} W n$ cubic hypermatrices $\left\{H W_{\text {symmetric }}\right\}$ and $\left\{H T_{\text {symmetric }}\right\}$, e.g. the symmetric elements of the semisimple sub-algebra, see table 2, generate a set of cubic semisimple hypermatrices, by symmetry we mean $W^{t}=W$ in all hypermatrix plane directions. In short, therefore, we obtain the following rule: $\left\{\left(W S_{\text {symmetric }}, W T_{\text {symmetric }}\right)\right\}=\left\{W_{\text {trace zero algebra }}\right\}$. The generalization to any size components matrices and hypermatrices follows by induction on dimension.

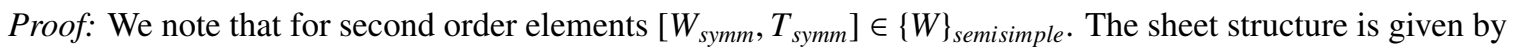

$$
\left.\left(W_{s}, W_{t}\right)=\left(\begin{array}{l}
S_{2} \\
S_{1}
\end{array}\right),\left(\begin{array}{l}
T_{2} \\
T_{1}
\end{array}\right)\right)=\left(\begin{array}{lll}
\left(S_{1}, T_{2}\right) & \left(S_{2}, T_{1}\right) & \\
& \left(S_{1}, T_{1}\right) & \left(S_{2}, T_{2}\right)
\end{array}\right)-\left(\begin{array}{ll}
\left(T_{1}, S_{2}\right) & \left(T_{2}, S_{1}\right) \\
& \left(T_{1}, S_{1}\right)
\end{array} \quad\left(T_{2}, S_{2}\right)\right)=\left\{W_{\text {ss }}\right\}_{\text {algebra }},
$$

ss-semisimple

and the product hypermatrix is composed of symmetric sheet elements generated through the product of symmetric hy- 
permatrices $W_{s}$ and $T_{s}$. Since the hypermatrices are symmetric and the sheets are identical and have trace zero the product $\left(W_{s}, W_{t}\right)$ and its commuting product $\left(W_{t}, W_{s}\right)$ must have trace zero, and since the product is generated by subtracting the two resulting symmetric hypermatrices the product must be a trace zero hypermatrix, see product illustration. Here $\mathrm{S}$ is -a cubic symmetric hypermatrix element, and T- is a cubic symmetric hypermatrix element of the Lie algebra. Hence, the Lie bracket product of cubic symmetric hypermatrices with symmetric sheet structure with another set of cubic symmetric hypermatrices with symmetric sheets structure generates a trace-zero hypermatrix set.

\subsection{Semi-skew-symmetric hypermatrices}

Definition 5 A semi skew symmetric hypermatrix has the following property: $W=-W_{\otimes}$, $(\otimes$ : skew-symmetric sheet interchange in at least one plane direction). Looking at the components of table 2 we find that semi-skew-symmetric trace zero sub-algebra generates a semisimple set of hypermatrices.

For generating elements of order 2 and above in higher hypermatrix algebra we have the following rule: Semi skew symmetric trace zero sub-algebra generates semi-simple algebraic Sets.

From table 2 we infer that the semisimple algebra $\left\{W_{S}\right\}$ splits ambiguously $\left\{W_{S}\right\}=W_{S: T z} \oplus W_{S, \text { nonTz }} \oplus W_{S: s k s}$. (Tz: trace zero, sks: skew-symmetric). The set $\left\{W_{S}\right\}$ and the multiplicative elements of $\left\{W_{S}\right\}$ in the set $\left\{t_{2}, t_{3}, t_{4}, t_{6}, t_{7}, t_{8}\right\}$ generates an infinite set. It also splits, ambiguously.

$$
W_{S \infty}^{\infty}=W_{S T z}^{\infty} \oplus W_{\text {TnonTz }}^{\infty} \oplus W_{T: S y m m e t r i c}{ }^{\infty} \oplus W_{T: S K S}{ }^{\infty} .
$$

The algebraic sets generated from $\left\{W_{S} /\left\{t_{1}, t_{5}, t_{9}\right\}\right\}$ is a semisimpe Lie hypermatrix algebra. The multiplicative Lie set $\left\{t_{2}, t_{3}, t_{4}, t_{6}, t_{7}, t_{8}\right\}$ carries periodic properties with respect to its fundamental trace zero elements and sheet structure, some of the infinite properties and periodic properties will be discussed here (Cooke, 1955; Kac, 1990).

What are the periodic properties of $W_{T \infty}{ }^{\infty}$ Lie algebra?

A fixed algebra in $\left\{W^{n^{*}}\right\}$ algebra is a subalgebra of $\{W\}$ that does not change properties as we generate each level Lie algebra, although, hypermetrics may change: size and the number of sheets in the hypermatrix and representation varies. If $W_{s}$ is a sub algebra of $\mathrm{W}$ we write for the fixed subalgebra $f^{n}\left(W_{s}\right)=W^{*} W=W^{*} s, \forall n$. For the semisimple WT algebra the fixed stable set is given by $\left\{t_{1}, t_{5}, t_{9}\right\}$ and we may write for all $\left.W^{n^{*}}, f^{n}{ }_{L i e}\left(W_{\left\{t_{1}, t_{5}, t_{9}\right\}}\right)=W^{n^{*}} t_{1}, t_{5}, t_{9}\right\}$.

Theorem $2 f^{n}{ }_{L i e}\left(W_{\left\{t_{1}, t_{5}, t_{9}\right\}}\right)=\alpha W_{\left\{t_{1}, t_{5}, t_{9}\right\}}^{s_{1}}, \forall n, \alpha$ is a structure constant.

Proof. The proof is by induction and induction on dimension. For $W_{2 \times 2 \times 2}$ we have, reading the symmetric elements from table 2

$<$ Table 4>

The next set of elements for $W(t)_{2 \times 2 \times 4}$ are given by $2 t^{*}{ }_{1},-2 t^{*}{ }_{1}, 2 t^{*}{ }_{5},-2 t^{*}{ }_{5}, t^{*}{ }_{9}$, and all these elements will differ only by a structure constant and the number of sheets when extended with respect to the basis $\left\{t_{1}, t_{5}, t_{9}\right\}$. Similarly for $W(T)_{2 \times 2 \times 4 n}$ the set $\alpha t^{*}{ }_{1},-\alpha t^{*}{ }_{1}, \alpha t^{*}{ }_{5},-\alpha t^{*}{ }_{5}, \alpha t^{*}{ }_{9}$ stay fixed under algebraic extensions of the basis elements.

From table 2 and 3 and from theorem-2 I conjecture as follows:

Conjuncture 1 There exists a semisimple Lie subalgebra that stay's fixed under all algebraic extensions. From table-4 I conjecture:

Conjuncture 2 There exists a non trivial semisimple Lie sub-algebra not containing the center of W that stay's trace zero algebra under all algebraic extensions. In fact I will show that there exists more then one such semisimple extension of the basis elements.

In table 2, the semi-skew-symmetric elements (only the hypermatrices are skew-symmetric) of $\left\{W_{\text {semisimple }}\right\}$ are given by:

$$
\begin{array}{r}
\left(t_{4}, t_{2}\right)=\left(\begin{array}{ccc} 
& 0 & \\
0 & & h \\
& -h &
\end{array}\right) \\
\left(t_{7}, t_{3}\right)=\left(\begin{array}{ccc}
-h & & 2 x \\
& -2 x &
\end{array}\right) \\
\left(t_{8}, t_{6}\right)=\left(\begin{array}{ccc}
I & & \\
-I & & 2 y
\end{array}\right)
\end{array}
$$

These hypermatrices have only global skew-symmetry in one transposed plane direction because the constituent sub- 
matrices are not skew-symmetric, in fact the first two are trace zero elements and the third has non trace-zero elements yet the sum total of the diagonal elements for the hypermatrix is zero, global trace-zero. Skew symmetric hypermetrics of order 2 are generated by pairs of anti-symmetric hypermatrices as follows:

2.5.1 Anti-symmetric hypermatrices with nilpotent components

$$
\left(t_{4}, t_{2}\right)=\left[\left(\begin{array}{l}
y \\
x
\end{array}\right),\left(\begin{array}{l}
x \\
y
\end{array}\right)\right]
$$

2.5.2 Anti-symmetric hypermatrices with nilpotent and symmetric components

$$
\left(t_{8}, t_{6}\right)=\left[\left(\begin{array}{l}
h \\
y
\end{array}\right),\left(\begin{array}{l}
y \\
h
\end{array}\right)\right]
$$

Generally: $\left(W_{f(h, y, x)}, W_{\otimes f(h, y, x)}\right)=W^{*}$ trace zero hypermatrix

$W^{*}$ is a product of trace zero, nilpotent elements in anti symmetric hypermetrics.

$\otimes$-anti-symmetric arrangement of any cubic sheet arrangement of the basis elements $f(h, y, x)$. For example $(h, y) \rightarrow$ $(y, h),(h, y, x) \rightarrow(y, h, x),(h, y, x, 5 h-x) \rightarrow(y, h, 5 h-x, x)$ etc'.

In conclusion we may sum up with rule 2 stating that:

$$
\left\{\left(W H_{f(h, y, x)}, W H_{\otimes f(h, y, x)}\right)\right\}=\left\{W H_{\text {semi skew-symmetric hypermatrix algebra }\}}\right.
$$

The Lie product of hypermetrics (composed of h, y, x elements) set in anti-symmetric hypermatrices, result in skewsymmetric trace zero hypermatrices, or semi skew-symmetric hypermatrices. The generalization to any size hypermatrices follows by induction on dimension.

\section{Third Extension of Lie Hypermatrix Algebra}

If the hypermatrices are skew-symmetric we denote the semi-skew-symmetric elements in table 2 as follows: $\left(t_{4}, t_{2}\right)=$ $t_{4,2},\left(t_{7}, t_{3}\right)=t_{7,3},\left(t_{8}, t_{6}\right)=t_{8,6}$.

The next cubic extension is a 16 sheet hypermatrix cube skew sub-algebra set in the 4-dimensional cube and it is expressed here by the bracket notation:

$$
\left(t_{4,2}, t_{8,6}\right)=(2 y, h,-h,-2 y,-h, 0,0, h, h, 0,0,-h,-2 y,-h, h, 2 y)
$$

The product is read from the right to the left; it could be set clockwise in an hypermatrix setting.

Thus, if we take the Lie product of hypermatrices composed of identity sub-matrices trace zero sub-matrices and nilpotent sub-matrices, the resulting Lie products is a set of trace zero hypermatrices. Generally we have:

$$
\text { (Semi Skew - Symmetric, Semi Skew - Symmetric) } \rightarrow \text { Trace zero hypermatrix. }
$$

For example, we have: $\left(t_{4,2}, t_{7,3}\right)=(2 x, I,-I,-2 x,-I, 0,0,-I,-I ., 0,0,-I,-2 x,-I, I, 2 x)$

Semi Skew - Symmetric $\times$ Symmetric $($ Nonzero trace,Trace zero) - Symmetric $($ Nonzero Trace, Trace zero $) \times$ Semi Skew - Symmetric $\rightarrow \pm$ identity, \pm idempotent, nonzero trace. More examples.

For example: $\left(t_{7,3}, t_{8,6}\right)=(-h, 2 x+2 y,-2 x-2 y, 4 h, 2 y-2 x, 0,0,2 y+2 x, 2 y+h, 0,0,-2 y-2 x, 4 h,-4 x-2 y, 2 x-4 y,-4 h)$

The product is read from the right to the left. The Lie bracket hyper-products of hyper-matrices with sheet skew symmetry or sheet nilpotent hyper-matrices or sheet identity hyper-matrices results in trace zero hypermatrices.

Skew sub-algebra could be extended skew-symmetrically in 4 -cubic-hypermatrices, $n=1,2,3, \ldots$ In that case what would be the next skew symmetric sub-algebra?

In general if $\mathrm{W}$ is semi skew-symmetric hypermatrix with respect to sheets of matrices we have $t_{x, y}=-t_{y, x}$, more generally $t_{x i}=-t_{\otimes x i}$.

If the components are trace zero: semisimple and skew symmetric (e.g., the basis element are h or $\alpha \times h$ ) then semisimplicity is maintained when extended by Lie brackets product. We may sum up these ideas of generating a semisimple Lie hypermatrix algebra from skew symmetric components as follows:

Theorem 3 (symmetric, skew-symmetric and anti-symmetric hypermatrix generators) The semisimple symmetric, skewsymmetric hypermatrix sub-algebra $\left\{W_{\text {symmetric/skewsymmetric }}\right\} \in S l_{2} W n$ associated with the Lie algebra $\left\{W_{n}\right\}$ could be extended dynamically in cubic dimension $4^{n}, n=2,3, \ldots$. It generates a semi periodic symmetric, skew symmetric sets in 
higher dimension cubes when, for example, it operates anti-symmetrically on symmetric, skew-symmetric hypermatrices elements of $\left\{W_{n}\right\}$ producing $\left\{W_{\text {semisimple }}\right\}$ - Lie sub-algebras.

Proof. We note that the second order elements $\left[W_{\text {skewsymmetric }}, T_{\text {skewsymmetric }}\right] \in W_{\text {semisimple }}$ is an inclusion for certain products. The sheet structure for $\left(W_{s k s}, W_{s k s}\right)$ is given by

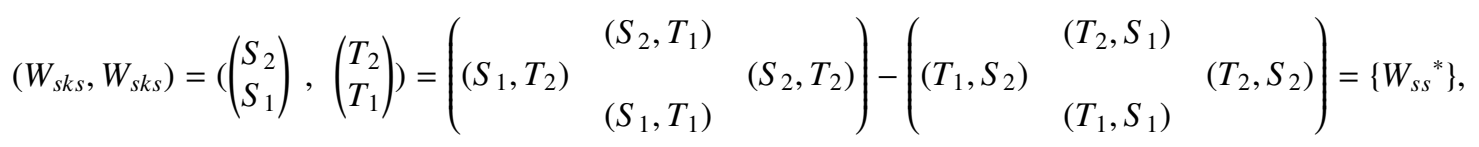

\section{Sks-skewsymmetric}

and the product hypermatrix is composed of skewsymmetric hypermatrices with antisymmetric sheet elements generated through the product of skewsymmetric hypermatrices $W_{s k s}$ and $T_{s k s}$. Since the hypermatrices are skewsymmetric in the same directional plane and the hypermatrix sheets are trace zero sheets and have antisymmetric sheet arrangement the components product elements of $\left(W_{s k s}, W_{s k s}\right)$ must have trace zero, and since the product is generated by subtracting the products of two anti-symmetric hypermatrices the resulting product must be a trace zero hypermatrix. Here $\mathrm{S}$ and $\mathrm{T}$ are symmetric hypermatrix element of the Lie algebra.

Trivially, if the components sheets are skew symmetric and they commute, see for example the elements along the diagonal of table $2, t_{4,2}, t_{8,6}, t_{7,3}$, then arranging them anti-symmetrically will suffice for generating trace zero elements.

Theorem 4 The bracket product of trace zero $\mathrm{Sl}_{2} \mathrm{~W}_{n}$ nilpotent hypermatrices of the same type and nilpotent hypermatrices of the same type which possibly include identity matrices and no repeating sub-hypermatrices, generate a semisimple hypermatrix Lie algebra.

Proof. If the set from which we generate hypermatrices is composed of matrix elements that are of the same nilpotent type say $x$ or $y$ denoted $N_{p}$, and possibly an identity matrix in the set of hypermatrices the bracket product will generate a trace-zero element.

$[\{\text { Nilpotent, or } \pm I\},\{\text { Nilpotent, or } \pm I\}]_{\text {identical nilpotent elements up to product }} \rightarrow$ Semisimple algebra.

For example $\left(t_{6,9}, t_{5,8}\right)=(0,0,0,0,0,0, y, 0,0,-y, 0,2 y, 0,0,-2 y, 0)$.

$\left[f\left\{N^{\Delta}, I^{\Delta}\right\}, f\left\{N^{\Delta}, I^{\Delta}\right\}\right] \Longrightarrow$ Semisimple algebra.

$\Delta$-Indicates possible omission of $N_{p}$ or I elements.

The left Lie product is either zero, or nilpotent and the right product is either zero or nilpotent since either product is I or $-I$ and not both the same the sum of the diagonal elements will be zero.

\section{References}

Bourbaki, N. (1980). Elements of mathematics: Lie groups and Lie algebras: Chapters 1-3. Springer-Verlag.

Cayley, A. (1843). In Muir Thomas, Metzler William 1933. A Treatise on the theory of Determinants. Dover publication New York, pp. 745-756.

Cooke, Richard G. (1955). Infinite matrices and sequence spaces, Dover pub.

Humphreys, E. James. (1972). Introduction to Lie algebra and representation theory. Springer-Verlag.

Kac, Victor G. (1990). Infinite-dimensional Lie algebras. Third edition. Cambridge University Press.

Serre, J-P. (1987). Complex semisimple Lie algebras. Springer-Verlag.

Table 1. Trace zero elements of order 1

\begin{tabular}{|c|c|c|c|}
\hline$()$, & $\mathrm{X}$ & $\mathrm{Y}$ & $\mathrm{h}$ \\
\hline $\mathrm{X}$ & 0 & $\mathrm{~h}$ & $-2 \mathrm{X}$ \\
\hline $\mathrm{Y}$ & $-\mathrm{h}$ & 0 & $2 \mathrm{Y}$ \\
\hline $\mathrm{h}$ & $2 \mathrm{X}$ & $-2 \mathrm{Y}$ & 0 \\
\hline \multicolumn{4}{|c|}{} \\
\multicolumn{4}{|c|}{ symmetric, $x, y-$ nilpotent }
\end{tabular}


Table 2. Generating $W_{\text {trace zero elements }}$ and the structure of $S u_{2} W_{4}$

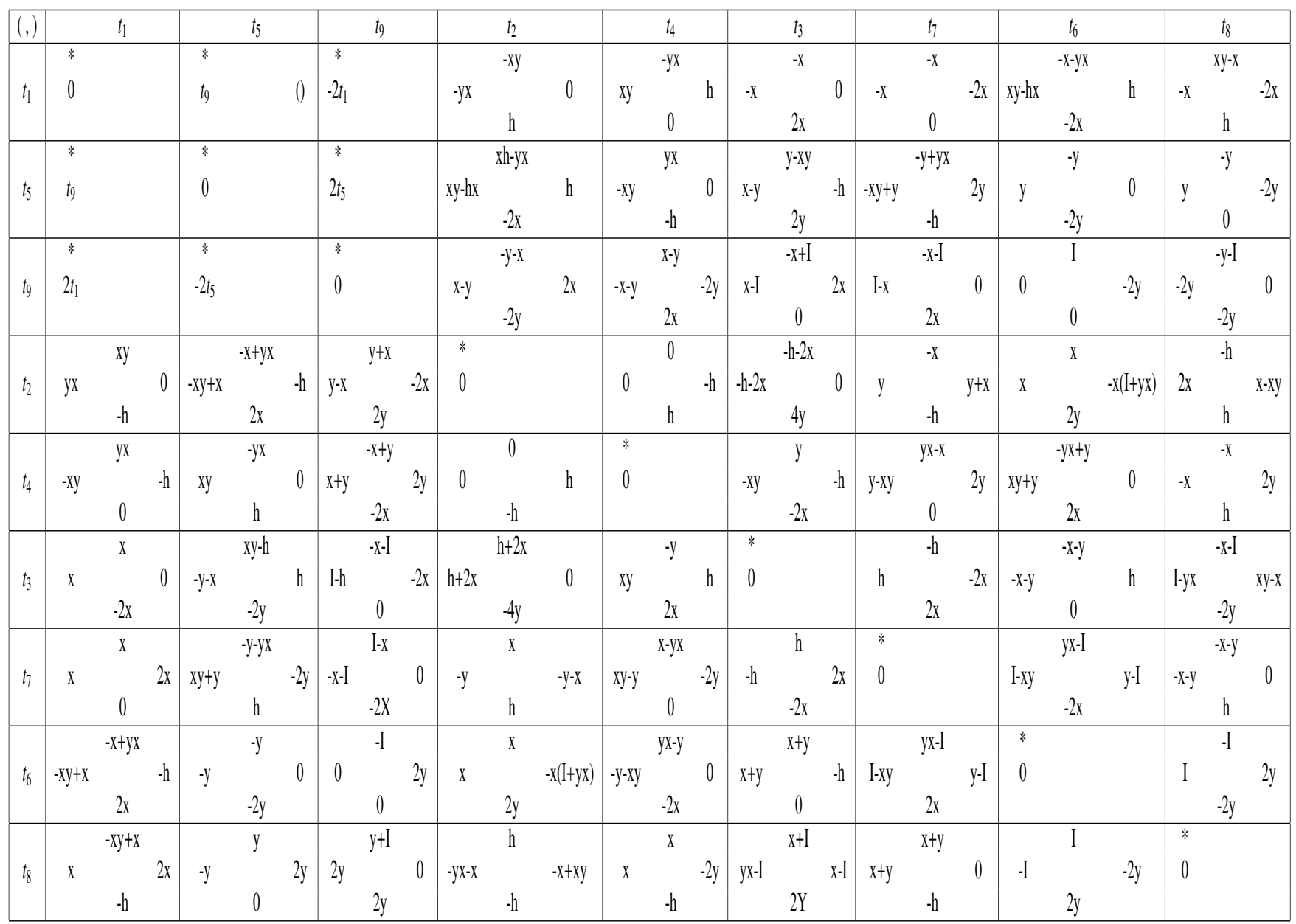

In table 2 there are trace zero symmetric and skew-symmetric hypermatrices, some of the skew symmetric hypermatrices are skewed in only one plane/direction of sheet interchange, i.e., $W^{t}=-W_{\otimes}$, and off the main diagonal there are nonzero trace hypermatrices with products such as $h h=I, x h-y x$, see definitions for $x y$ and $y x$ below. Nine upper left corner hypermatrices entries constitute a symmetric trace zero-central semisimple sub-Lie algebra with symmetry in only one plane direction, globally for that set of hypermatrices $W^{t}=W .(\otimes$ : stands for symmetric sheet interchange in at least one plane direction).

Table 3. Multiplication (left to right) table for trace zero elements of order 1

\begin{tabular}{|c|c|c|c|}
\hline$\rightarrow \mathrm{x}$ & $\mathrm{x}$ & $\mathrm{y}$ & $\mathrm{h}$ \\
\hline $\mathrm{x}$ & 0 & $1-\mathrm{yx}$ & $-\mathrm{X}$ \\
\hline $\mathrm{y}$ & $1-\mathrm{xy}$ & 0 & $\mathrm{Y}$ \\
\hline $\mathrm{h}$ & $\mathrm{X}$ & $-\mathrm{Y}$ & $-\mathrm{I}$ \\
\hline
\end{tabular}

Table 4. Symmetric elements

\begin{tabular}{|c|c|c|c|}
\hline$(,)$, & $t_{1}$ & $t_{5}$ & $t_{9}$ \\
\hline$t_{1}$ & $*$ & $*$ & $*$ \\
& 0 & $t_{9}$ & $-2 t_{1}$ \\
\hline$t_{5}$ & $*$ & $*$ & $*$ \\
& $t_{9}$ & 0 & $2 t_{5}$ \\
\hline$t_{9}$ & $*$ & $*$ & $*$ \\
& $2 t_{1}$ & $-2 t_{5}$ & 0 \\
\hline
\end{tabular}




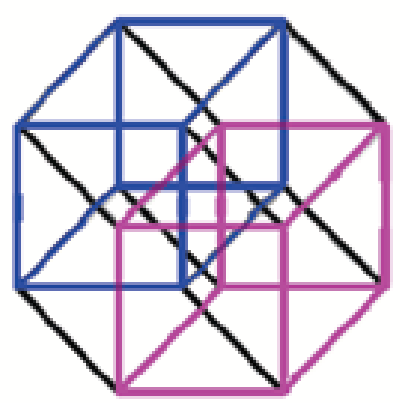

Figure 1 